\title{
The emergence of enterovirus D68 in England in autumn 2014 and the necessity for reinforcing enterovirus respiratory screening
}

\author{
A. I. CARRION MARTIN*1,2, R. G. PEBODY ${ }^{2}$, K. DANIS ${ }^{1,3}$, J. ELLIS $^{2}$, \\ S. NIAZI ${ }^{2}$, S. DE LUSIGNAN ${ }^{4,5}$, K. E. BROWN ${ }^{2}$, M. ZAMBON ${ }^{2}$ AND \\ D. J. ALLEN ${ }^{2,6} \dagger$ \\ ${ }^{1}$ European Program for Intervention Epidemiology Training (EPIET), European Centre for Disease Prevention \\ and Control, (ECDC), Stockholm, Sweden \\ ${ }^{2}$ Public Health England (PHE), London, UK \\ ${ }^{3}$ French Institute for Public Health Surveillance (Institut de Veille Sanitaire, InVS), Paris, France \\ ${ }^{4}$ Royal College of General Practitioners (RCGP) Research and Surveillance Centre (RSC), Euston, London, \\ $U K$ \\ ${ }^{5}$ University of Surrey, Guildford, $U K$ \\ ${ }^{6}$ NIHR Health Protection Research Unit in Gastrointestinal Infections, Colindale, UK
}

Received 3 May 2016; Final revision 30 January 2017; Accepted 1 March 2017; first published online 3 April 2017

\section{SUMMARY}

In autumn 2014, enterovirus D68 (EV-D68) cases presenting with severe respiratory or neurological disease were described in countries worldwide. To describe the epidemiology and virological characteristics of EV-D68 in England, we collected clinical information on laboratoryconfirmed EV-D68 cases detected in secondary care (hospitals), between September 2014 and January 2015. In primary care (general practitioners), respiratory swabs collected (September 2013-January 2015) from patients presenting with influenza-like illness were tested for EV-D68. In secondary care 55 EV-D68 cases were detected. Among those, 45 cases had clinical information available and $89 \%$ (40/45) presented with severe respiratory symptoms. Detection of EV-D68 among patients in primary care increased from $0.4 \%$ (4/1074; 95\% CI $0.1-1.0)$ (September 2013-January 2014) to $0.8 \%$ (11/1359; 95\% CI 0.4-1.5) (September 2014-January 2015). Characterization of EV-D68 strains circulating in England since 2012 and up to winter 2014/2015 indicated that those strains were genetically similar to those detected in 2014 in USA. We recommend reinforcing enterovirus surveillance through screening respiratory samples of suspected cases.

Key words: Enterovirus D68, neurologic manifestations, respiratory infections, respiratory samples.

\footnotetext{
* Author for correspondence: A. I. Carrión Martín, National Infection Service, Public Health England, 61 Colindale Avenue, London, UK

(Email: Isidro.carrion@phe.gov.uk)

$\dagger$ Present address: Pathogen Molecular Biology Department, Faculty of Infectious and Tropical Diseases, London School of Hygiene \& Tropical Medicine, London, UK.
}

\section{BACKGROUND}

The Enterovirus genus (family Picornaviridae) includes 12 species (A-J) of which four (A-D) are associated with disease in humans; each of these species encompasses multiple types. Enterovirus infection can be associated with a broad range of clinical presentations, including meningitis, encephalitis, hand-foot-andmouth disease (HFMD), respiratory illness, and more rarely, neurological symptoms. Multiple enterovirus 
types co-circulate, and some types are known to emerge occasionally in a population to cause outbreaks or clusters of cases of acute neurological disease [1, 2]. Increasing recognition of the disease burden associated with enterovirus infections, and the description of 're-emerging' enteroviruses such as EV-C99, EV-C104, EV-D70, EV-A71 [3, 4], provide evidence that enteroviruses have rapidly evolving genomes, are ubiquitous worldwide, spread rapidly via faecal-oral and environmental routes, but also via respiratory transmission, and have the ability to cause severe disease. Enterovirus D68 (EV-D68) is one of five enterovirus types assigned to Enterovirus species D. The virus was first described in 1962, isolated from children with pneumonia and bronchitis [5]; in 2002 a human rhinovirus 87 was reclassified as EV-D68, based on phylogenetic analysis [6]. In countries with enterovirus established surveillance EV-D68 has been detected sporadically in cases of acute respiratory illness, particularly in children, and occasionally was associated with acute neurological disease and other severe outcomes [7, 8].

In autumn 2014, the USA experienced a nationwide outbreak of EV-D68 associated with severe acute respiratory illness [9]. This outbreak coincided with an apparent increase in incidence of reported cases of neurological disease characterized as acute flaccid myelitis (AFM), suggesting that AFM is a rare yet severe clinical manifestation of EV-D68 infection [10-12].

In 2014, following the USA outbreak, EV-D68 was detected in European countries [13]. In Norway, EV-D68 was detected in $11 \%$ of 303 paediatric nasopharyngeal samples collected from children hospitalized with acute respiratory infection. EV-D68 was associated with acute flaccid paralysis in one child [14]. In the Netherlands, 1\% (18/1896) of respiratory samples taken from patients with respiratory symptoms tested positive for EV-D68 [15]. Phylogenetic analysis showed that strains detected in the Netherlands were genetically similar to those circulating in the USA in 2014 [15].

Public Health England (PHE) has an established national Enterovirus Surveillance System (ESS), with emphasis on further investigation of enteroviruspositive cases presenting with acute neurological symptoms as part of enhanced poliovirus surveillance [16]. Following reports of the emergence of EV-D68 in USA, PHE issued guidance on October 2014 that EV-D68 should be considered as a possible cause of disease, particularly in children presenting with severe acute respiratory infections and/or unexplained neurological symptoms, and where standard respiratory virus screens were negative, or if a combined rhinovirus/enterovirus positive result was initially detected.

This study aimed to quantify the circulation of EV-D68 in England in primary and secondary care before and after the identification of EV-D68 in North America in autumn 2014. Our objectives were (i) to determine whether there was an increase in number of cases between autumn/winter 2013-2014 and autumn/winter 2014-2015, and (ii) to describe the clinical presentation and phylogenetic characteristics of this virus, including severity in England in the autumn/winter 2014-2015.

\section{METHODS}

A confirmed case was any patient presenting to primary or secondary care with a specimen positive for EV-D68 detected initially by one-step real-time reverse transcription PCR (rRT-PCR) [15]. Cases investigated in this study were identified from two surveillance sources.

\section{Secondary care (clinical presentations)}

As part of the ESS, samples (including stool, cerebrospinal fluid (CSF) and respiratory samples) from individuals who presented to hospitals with acute neurological syndromes and in whom an enterovirus is detected are sent for enterovirus typing. This typing is carried out at the Virus Reference Department, PHE, Colindale. From November 2014, referrals to the ESS were enhanced, to include enteroviruspositive paediatric cases with acute respiratory symptoms. The Respiratory Diseases Department of the PHE Centre for Infectious Disease Surveillance and Control (CIDSC) coordinated the epidemiological investigation of each confirmed case.

Using a standardized questionnaire the treating clinicians collected information on all confirmed EV-D68 cases from October 2014 to February 2015. The information gathered included: patient characteristics, clinical presentation (including details on the neurological and respiratory presentations), testing performed (including all pathogens detected and type of samples), and disease outcome.

\section{Primary care (EV-D68 circulation)}

To estimate the rate of circulation of EV-D68 in the community, we tested respiratory samples from the 
general practitioner (GP) influenza sentinel surveillance system. Patients presenting with acute febrile respiratory illness considered by their GP to be influenza-like illness (ILI) had nasopharyngeal virology specimens sent to PHE, as part of the Royal College of General Practitioners Research and Surveillance Centre (RCGP RSC) influenza sentinel surveillance weekly returns service. These specimens were tested for EV-D68. This service, run by RCGP RSC since 1957, provides clinical surveillance data from a sentinel network of over 100 general practices (GP) (primary health care centers) together with virological respiratory specimens, from a subset of patients presenting with ILI across England [17]. Each GP sends around five combined nose and throat swabs per week from patients consulting with ILI, particularly during the winter season, from around week 40 of one year to around week 20 of the next year. Among the samples received from the RCGP RSC from week 39 of 2013 to week 15 of 2015 (September 2013 to April 2015) only those testing negative for influenza virus were tested for EV-D68.

\section{Data analysis}

Changes in circulation of EV-D68 were estimated by calculating EV-D68 percent positivity in two periods. Percent positivity was calculated as number of EV-D68 positive samples divided by total of samples tested for EV-D68 in each period. Percent positivity in the period from week 39 of 2013 to week 05 of 2014 (September 2013 to January 2014) (in 2013 RCGP RSC influenza testing only started in week 39) was compared with percent positivity in the period from week 36 of 2014 to week 05 of 2015 (September 2014 to February 2015), using $\chi^{2}$ test. For primary care samples, we only looked at percent positivity and demographic characteristics (age and sex) of cases.

\section{Laboratory detection and characterization}

Detection of EV-D68 was performed by rRT-PCR as previously described [15]. Further characterization of EV-D68 strains was performed by amplification and partial sequencing of the VP1 coding region using a previously developed in-house assay (M. IturrizaGómara, University of Liverpool, UK). Briefly, primers Ent68F [5'-GAAGCCATACAAACTCGCAC-3'] and Ent68R [5'-ATTWGCAATGCTCATGTATGG-3'] were used to amplify a $~ 670$ nt fragment of the VP1 coding region. A high-fidelity PCR system was used (Expand
High Fidelity PCR Kit, Roche, UK) according to manufacturer's instruction, with the following specifications (per reaction): magnesium chloride final concentration $2.5 \mathrm{mM}, 40$ pmol each primer, and 1.75 U DNA polymerase. Amplification was performed under the following conditions: initial denaturation: $95^{\circ} \mathrm{C}, 240 \mathrm{~s}$; thermal cycling: $95^{\circ} \mathrm{C}, 30 \mathrm{~s}-42^{\circ} \mathrm{C}, 60 \mathrm{~s}-72^{\circ} \mathrm{C}, 60 \mathrm{~s}$ for 40 cycles; final extension: $72^{\circ} \mathrm{C}, 240 \mathrm{~s}$.

Sequence analysis was performed using Bionumerics v6.1 (Applied Maths, Kortijk, Belgium) and phylogenetic and molecular evolutionary analyses were conducted using MEGA version 6 [18].

For phylogenetic analysis, we included representatives of EV-D68 strains detected in the USA in 2014 [19] and 2015 [20], EV-D68 strains detected in England in 2010-2013 as part of national enterovirus surveillance and enhanced poliovirus surveillance [21] and representatives of other EV-D68 strains reported globally [22-24].

\section{RESULTS}

\section{Clinical presentation of EV-D68 in England}

From week 42 of 2014 to week 05 of 2015 (October 2014 to February 2015), 433 respiratory samples were collected from patients presenting to secondary care in England. During this period, 13\% (55/433) of these respiratory samples were positive for EV-D68, compared with $11 \%(3 / 26)$ of EV-D68 positive samples detected in the same period in the previous year (Fig. 1). Sixty-nine percent (38/55) of the EV-D68 cases (October 2014 to February 2015) were in children under 12 years of age; the median age of cases was 4 years (range $0-71$ ), and there was no difference in the gender distribution (46\% male) (Fig. 2). The majority of these EV-D68-positive specimens were nasopharyngeal aspirates, 44\% (24/55), and nose/ throat swabs, $31 \%(17 / 55)$. The remaining 14 were sputum, mouth swabs and tracheal/bronco alveolar aspirates. EV-D68 was not detected in any CSF specimen tested $(n=562)$.

Detailed clinical information was available for $82 \%$ (45/ 55) EV-D68 cases (Table 1). None of these cases reported having recently traveled outside of the UK. Ninety-one percent (41/45) of the cases presented with acute respiratory symptoms, one case presented with HFMD, one case had leukemia (respiratory symptoms not reported) and two cases presented with acute neurological symptoms. These cases showing neurological presentations were in children $<5$ years of age: 


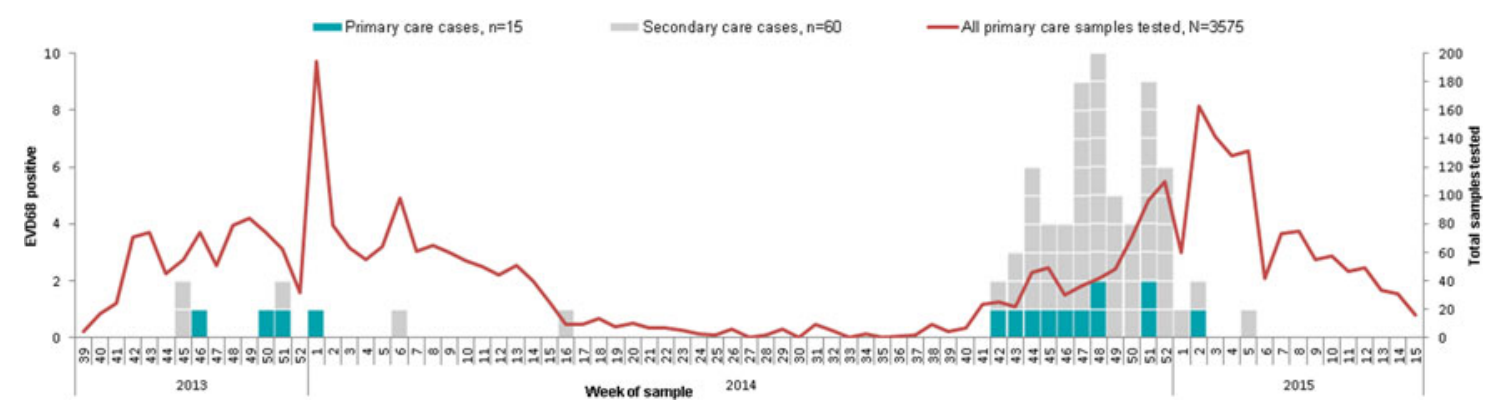

Fig. 1. Distribution of EV-D68 cases detected in the RCGP influenza sentinel surveillance system and in the ESS (including positives from before September 2014 and other later cases for whom clinical information is not available) by week of sample, England, September 2013 to March 2015.

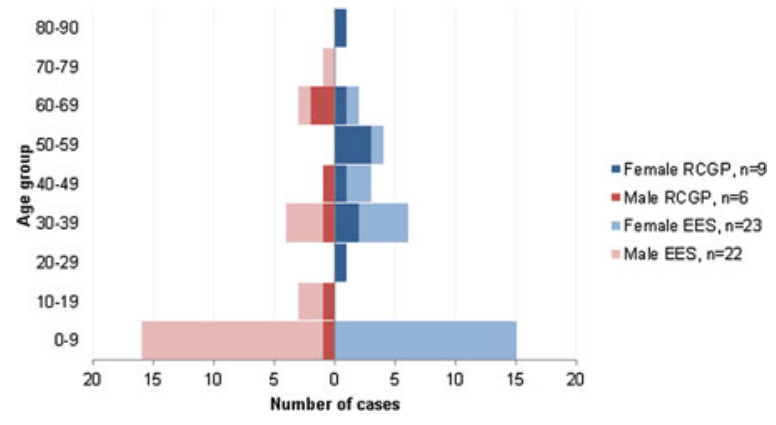

Fig. 2. Number of EV-D68 cases by sex, age, and surveillance system where detected, England, September 2013 to January 2015. (RCGP influenza sentinel surveillance system (primary care) and EES (secondary care)).

one case presented with meningoencephalitis and paucity of limb movements [25]; the second presented with afebrile seizures and respiratory arrest. These cases were previously healthy children who required intensive care unit (ICU) treatment and subsequently recovered; in both cases EV-D68 was detected in nasopharyngeal aspirate specimens. Thirty-three percent $(13 / 39)$ of the cases presented with asthma or wheezing, $84 \%(11 / 13)$ of them were children under 15 years of age (median age: 2 years) (information for asthma was only collected for 39 patients). Forty-seven percent (21/45) of the cases were immunocompromised or had an underlying chronic condition. Eighty-two percent (37/45) of the cases were admitted to hospital, with $27 \%(12 / 45)$ requiring treatment in ICU, all of them children (median age: $<1$ year) (Table 1). Two patients in whom EV-D68 was detected died: both were infants with underlying neurological problems. Information on cause of death was only accessible for one of them: it was likely due to complications in the underlying neurological problems. Two other infant cases had never left the hospital since birth.
Table 1. Clinical presentation and severity of EV-D68 positive cases in secondary care in England, September 2014 to January 2015

\begin{tabular}{lll}
\hline \hline $\begin{array}{l}\text { Clinical presentations- } \\
\text { severity }(N=45)\end{array}$ & $n$ & $\%$ \\
\hline $\begin{array}{l}\text { Respiratory symptoms } \\
\text { (including one case with }\end{array}$ & 41 & 89 \\
$\quad$ neurological symptoms) & 13 & $33(N=39)$ \\
$\begin{array}{l}\text { Asthma or wheezing } \\
\text { Immunocompromised or }\end{array}$ & 21 & 46 \\
$\quad \begin{array}{l}\text { severe underlying } \\
\text { diseases (all) }\end{array}$ & & \\
$\begin{array}{l}\text { Admitted to hospital } \\
\text { Respiratory } \\
\text { presentations }\end{array}$ & 37 & 82 \\
$\begin{array}{l}\text { Intensive care } \\
\text { Neurological } \\
\text { presentations }\end{array}$ & 12 & 75 \\
Deaths & 2 & 4 \\
\hline \hline
\end{tabular}

\section{Estimate of prevalence of EV-D68 in England}

A total of 3575 nose/throat swab samples were collected from week 39 of 2013 to week 15 of 2015 (September 2013 to April 2015) as part of the RCGP RSC influenza sentinel surveillance weekly returns service. The median age of the patients providing these samples was 33 years (range $0-99$ ), 17\% of them were children $<12$ years of age. Sixty-one percent were female.

EV-D68 was detected in $0 \cdot 4 \%(15 / 3575)$ of all the community respiratory samples analysed. Among the cases, the median age was 40 years, (range 5-84 years); nine $(60 \%)$ of them were female (Fig. 2). Among those 3575 samples, $2433(1074+1359)$ had been collected during the two periods of comparison and were included in the percent positivity analysis. Positivity in the autumn/winter 2013/14 (from week 
39 of 2013 to week 05 of 2014) was $0 \cdot 4 \%$ (4/1074; $95 \%$ CI $0 \cdot 1-1 \cdot 0)$. Positivity in the autumn/winter $2014 / 15$ (from week 36 of 2014 to week 05 of 2015) was $0 \cdot 8 \%(11 / 1359)(95 \%$ CI $0 \cdot 4-1 \cdot 5)(P=0 \cdot 17)$. All primary care (RCGP RSC) EV-D68 positive samples in 2013 were collected between weeks 40 and 47, while all the community EV-D68 positive samples from 2014 were collected between weeks 49 and 52 (Fig. 1).

\section{Phylogenetics}

Partial VP1 sequence was obtained from 88\% (57/70) of the EV-D68 cases identified from primary and secondary care in this study (four from community cases recruited via the RCGP network and 53 from cases referred to the ESS).

Of the 57 EV-D68 sequences detected in this study, 56 clustered with EV-D68 genetic clades (A and B as described elsewhere [26]): $49(85 \%)$ in clade B and 8 (14\%) in clade A. The remaining 2014 strain clustered with the prototype US/Fermon/1962 strain (Fig. 3).

EV-D68 strains detected in England in 2014 and 2015 clustered both with strains detected in the USA during the same period, and with strains circulating in England prior to 2014. Among the clade B viruses there were the two sequences found in cases with neurological presentation. Both of these sequences were highly similar to other co-circulating viruses from the same period.

Amino acid sequences were highly similar within clades, with most amino acid differences occurring in the $\mathrm{BC}$ and $\mathrm{DE}$ loops (data not shown). There were no specific amino acid changes in either the $\mathrm{BC}$ or DE loop, or elsewhere in the regions of VP1 sequenced that distinguished strains associated with neurovirulence from other EV-D68 strains detected.

\section{DISCUSSION}

We used two established surveillance systems for this study. From the national ESS data we described the burden of illness due to EV-D68 and the severity of clinical presentation in England. We used the influenza sentinel GP surveillance system to look at the changes in EV-D68 circulation in primary care. Clinical information from the cases identified in the ESS (secondary care) found that cases with EV-D68-positive samples were mainly presenting with severe respiratory symptoms in the autumn/ early winter 2014-2015. In primary care, retrospective testing of samples revealed a non-significant increase in circulation of EV-D68 in autumn/early winter 2014-2015 compared with the same period the previous year.

Our findings from secondary care suggest that, when detected, EV-D68 is most frequently associated with respiratory disease, usually in children, and more rarely with neurological disease. Previous studies presented comparable results [9, 27-29]. Further, in our study the majority of hospitalized cases presented with severe respiratory disease and one-third of those were admitted to ICU (all of them children) and required respiratory support. Our results on cases with severe respiratory presentations, mostly children, associated with EV-D68 infection were comparable to similar findings in the Netherlands and the USA $[9,15]$. We found that $33 \%$ of cases presented with asthma/wheezing compared with the $70 \%$ of cases presenting with these symptoms in the 2014 USA outbreak [27], this difference could be attributable to the small number of cases for whom we had information on asthma/wheezing. In our study, similarly to other studies, almost half of the patients admitted with respiratory disease were immunosuppressed or had severe underlying conditions [15, 30]. Our findings reinforce the importance of clinicians considering EV-D68 (and other enteroviruses) as one of the differential diagnoses of severe acute respiratory illness, particularly in children.

In the only two acute neurological presentations in our study, EV-D68 was not detected in the CSF of these children and the detection of EV-D68 in the respiratory samples may have been coincidental. However, both cases were previously healthy and no other infectious cause was identified for the disease. Therefore we cannot exclude the possibility of an association between EV-D68 and their severe acute presentations. Both children made a complete recovery. Severe neurological presentations associated with EV-D68 in previously healthy children with a preceding respiratory infection have been reported in different European countries: in Norway, two cases (Autumn 2014) [31]; a case in France (September 2014) [32], and two cases in Wales (UK) (January 2016) [33], EV-D68 was not identified in the CSF in any of these cases, but rather from upper respiratory tract samples. Similarly, we found that EV-D68 RNA was detectable in respiratory specimens, but that other sample types (e.g. CSF or stool) were not sensitive for detection of EV-D68. We were able to recover infectious EV-D68 virus by inoculation of permissive cell lines with RT-PCR-positive respiratory 


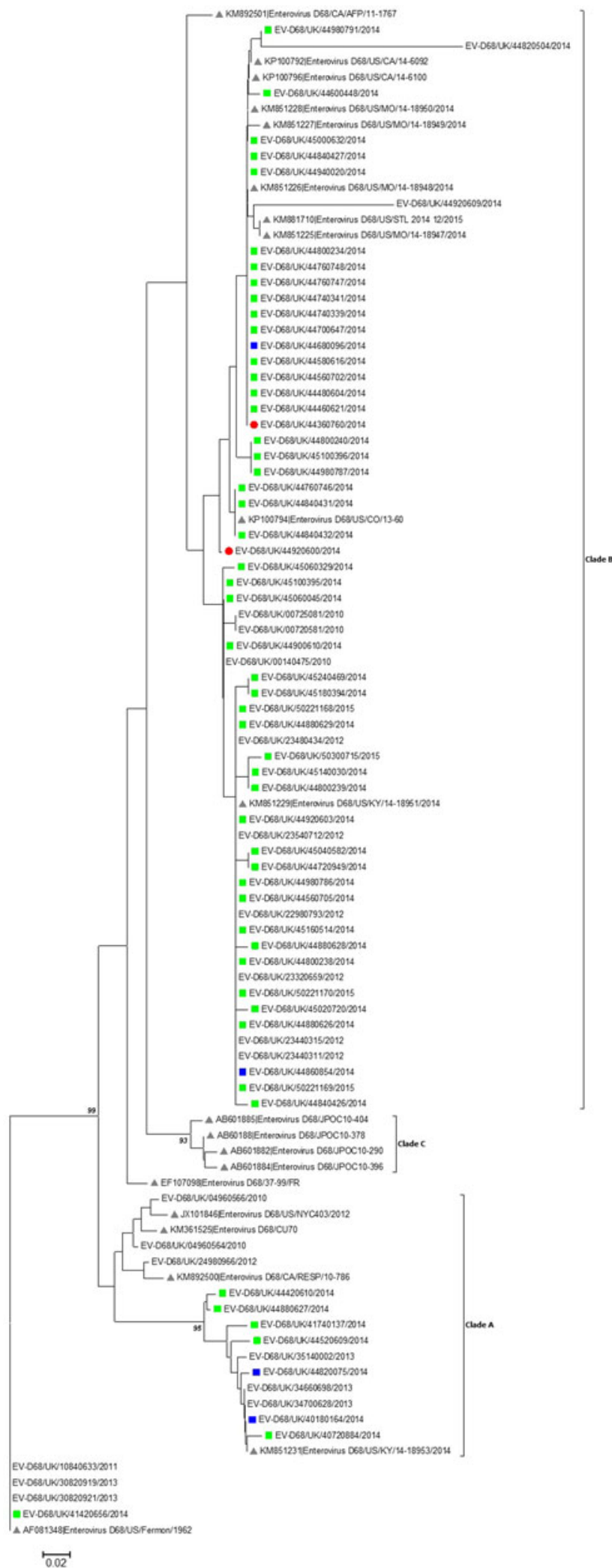

Fig. 3. Phylogenetic analysis of partial VP1 sequences of EV-D68 covering the BC and DE loops of the VP1 region. The evolutionary history was inferred using the Neighbour-Joining method and the optimal tree with the sum of branch length $=0.95104738$ is shown. The percentages of replicate trees in which the associated taxa clustered together in the bootstrap test (1000 replicates) are shown next to the branches (where $>90 \%$ ). The tree is drawn to scale. Analyses were conducted in MEGA6 [18]. = (green) = ESS specimens; $\mathbf{a}($ blue $)=$ RCGP specimens; $\bullet$ = Cases associated with neurological illness; $\Delta=$ Reference sequences. 
specimens, but not other specimen types. This highlights the importance of collecting all clinically relevant samples for differential diagnosis in such acute presentations and indicates that in EV-D68 suspected cases respiratory specimens are the most appropriate for diagnostic testing and virological characterization.

The rate of positivity in cases of acute respiratory disease presenting to primary care in England doubled from autumn/winter 2013-2014 to autumn/winter 2014-2015. Nonetheless this increase was not statistically significant and the number of cases and positivity rates were low. This suggests that the high number of severe cases observed in secondary care in the period post autumn 2014 is presumably explained by the change in case definition and enhanced case ascertainment. Similarly, in the Netherlands, a non-significant increase in the percentage of EV-D68 positives was observed from $2013(0 \cdot 3 \%)$ to $2014(0 \cdot 6 \%)$ among patients presenting to the GP [34]. In another similar study in Germany from 2014, a higher percentage (7.7\%) of EV-D68-positive samples from outpatients presenting with ILI, and/or acute respiratory infection was reported [30]. Our primary care cases had similar distribution across all age groups, but in those in secondary care, age was skewed toward infants and young children (Fig. 2). This could be related to the PHE guidance, suggesting considering EV-D68 as a possible cause of disease in children with severe respiratory symptoms. Or, taking into account similar results from other studies, it may demonstrate a different age profile of the EV-D68 cases presenting with severe symptoms in secondary care compared with the profile of the mild presentations in primary care.

We characterized EV-D68 strains circulating in England since 2012 and up to winter 2014-2015, among which we identified strains genetically similar to those detected in 2014 in USA. These findings are consistent with similar results from Netherlands and Germany $[15,30]$. These studies describe emergence of distinct genetic clusters, which reflects the continuous evolution of EV-D68 strains in circulation.

With extensive genetic variation in the EV-D68 genome, continuous collection of genomic data by representative surveillance systems is required to ensure that PCR-based detection methods are capable of detecting contemporary circulating viruses. Genotyping assays are generally based upon sequence analysis of the VP1 coding region, whilst primary detection assays usually target the 5 '-untranslated region of the genome. With recognized sequence variation in both regions, complete genome sequences representing contemporary circulating viruses is essential in assuring performance of molecular diagnostic and characterization assays.

However, to fully understand the clinical significance of detection of EV-D68 in patients, alongside genomic characterization, isolation of the virus, characterization by neutralization and measurement of virus-specific antibody titers is required. Integrating 'classical' virology approaches and molecular techniques will better establish links between infection and disease in patients, as well as provide valuable technical information for improving laboratory diagnostics, monitoring genetic drift in the virus genome which may result in degradation of PCR primer/ probe-binding sites. Some molecular assays have been found to exhibit cross-reactivity between EV-D68 and rhinovirus which can lead to falsepositive results. Furthermore, with reports of other emerging and novel enterovirus types [1, 2, 35], it is important to continually enhance the virological, genomic and epidemiological understanding of these viruses by maintaining up-to-date diagnostic and reference methods, and monitoring the factors determining local and temporal differences in atypical disease outbreaks due to enteroviruses.

The detection of EV-D68 worldwide in 2014-2015 may be the 'tip-of-the-iceberg' of circulation of EVD-68. EV-D68 is rarely associated with severe disease, which is probably why fewer cases have been detected by current surveillance systems that are designed to capture more severe (neurological) infections. In our study, detection of the virus in community and increased detection of the virus in hospitalized patients with respiratory disease suggest EV-D68, and perhaps other enteroviruses, are a more important cause of infection and, potentially, of disease than captured by current surveillance systems.

\section{STRENGTHS AND LIMITATIONS}

Our study had some strengths and limitations. In terms of strengths, we were able to look at the EV-D68 epidemiology and virology in primary care before and after the signal in the US, using an identical surveillance system, which allowed the comparison between the two periods with no ascertainment bias. In terms of weaknesses, first, we could not compare the EV-D68 positivity rate in ESS with previous years given the change in case definitions in autumn 2014 following the PHE guidance mentioned before. Second, the RCGP RSC influenza sentinel surveillance 
samples are predominantly restricted to the winter months when influenza is circulating, considering the previously described EV-D68 seasonal pattern, majority of cases occurring in late summer and autumn [36, 37], we may have missed cases if they had occurred in summer or late spring. Furthermore, the representativeness of the RCGP RSC influenza sentinel surveillance is not ideal for an estimate of the real circulation of this virus in patients presenting to primary care, as the patients tested present with acute febrile respiratory illness, and during the 2014 outbreak in the USA only $48 \%$ of the hospitalized cases presented with fever; additionally EV-D68 may be a more common cause of mild respiratory illness both in primary care and in the community among cases who self-manage without seeking healthcare intervention. Nonetheless, the RCGP RSC is a representative network, with only small differences with the national population and therefore the results of this study provide a useful baseline for future monitoring of the circulation of EV-D68 and its severity in England [17]. Finally, we did not sequence the whole genome which might contain virulence markers elsewhere. For example, changes in the 5'UTR and IRES have been proposed to associate with changes in translation initiation [22] and virulence [38, 39].

\section{CONCLUSIONS}

In this study we detected EV-D68 in cases of severe acute respiratory illness and, less commonly, in neurological illness, particularly in children and those with underlying disease from autumn 2014 when ESS was enhanced and respiratory sampling was included. We also provided evidence of EV-D68 in cases of ILI presenting in primary care before and after autumn 2014, with strains genetically similar to those detected in 2014 in the USA.

EV-D68 should be considered as a possible cause of disease in patients presenting with severe acute respiratory infections and/or with unexplained neurological symptoms. Our findings emphasize the necessity of reinforcing enterovirus surveillance in England. We recommend the screening of respiratory samples for enterovirus, particularly EV-D68, from patients presenting with severe acute respiratory infections and/or with unexplained neurological symptoms, when all other respiratory virus screen are negative or if an indeterminate rhinovirus/enterovirus positive result was initially detected. The surveillance of enterovirus, specifically EV-D68, in respiratory samples will help us to better understand the epidemiology of EV-D68 and to inform surveillance and laboratory-testing guidance.

\section{ACKNOWLEDGEMENTS}

The authors thank the patients who consented to virology specimens and the practices and hospitals that provided data for this study. This study was supported by Public Health England (PHE). The authors gratefully acknowledge the expert technical assistance of staff in the Enteric Virus Unit and Respiratory Virus Unit, Colindale, Public Health England (PHE). They acknowledge colleagues across PHE laboratories, NHS centers and GPs. They also acknowledge Hubert G.M. Niesters (University Medical Centre Groningen, NL) for making available PCR assay details ahead of publication. They are grateful to Nicholas W. Machin (Manchester Royal Infirmary, UK) for his assistance in obtaining clinical details. This research received no specific grant from any funding agency, commercial or not-for-profit sectors.

\section{DECLARATION OF INTEREST}

None.

\section{ETHICAL STANDARDS}

No ethical approval was required for this study.

\section{REFERENCES}

1. Horner LM, et al. Acute flaccid paralysis associated with novel enterovirus C105. Emerging Infectious Diseases 2015; 21(10): 1858-1860.

2. Osterback R, et al. Echovirus 30 meningitis epidemic followed by an outbreak-specific RT-qPCR. Journal of Clinical Virology: the Official Publication of the Pan American Society for Clinical Virology 2015; 69: 7-11.

3. Qiu J. Enterovirus 71 infection: a new threat to global public health? The Lancet Neurology 2008; 7(10): 868869.

4. Fischer TK, et al. Emergence of enterovirus $71 \mathrm{C} 4 \mathrm{a}$ in Denmark, 2009 to 2013. Eurosurveillance 2014; 19(38): pii. 20911.

5. Schieble JH, Fox VL, Lennette EH. A probable new human picornavirus associated with respiratory diseases. American Journal of Epidemiology 1967; 85(2): 297-310.

6. Ishiko H, et al. Human rhinovirus 87 identified as human enterovirus 68 by VP4-based molecular diagnosis. Intervirology 2002; 45(3): 136-141. 
7. Imamura T, et al. Enterovirus 68 among children with severe acute respiratory infection, the Philippines. Emerging Infectious Diseases 2011; 17(8): 1430-1435.

8. Kreuter JD, et al. A fatal central nervous system enterovirus 68 infection. Archives of Pathology \& Laboratory Medicine 2011; 135(6): 793-796.

9. Midgley CM, et al. Severe respiratory illness associated with a nationwide outbreak of enterovirus D68 in the USA (2014): a descriptive epidemiological investigation. The Lancet Respiratory Medicine 2015; 3(11): 879-887.

10. Greninger AL, et al. A novel outbreak enterovirus D68 strain associated with acute flaccid myelitis cases in the USA (2012-14): a retrospective cohort study. The Lancet Infectious Diseases 2015; 15(6): 671-682.

11. Messacar K, et al. A cluster of acute flaccid paralysis and cranial nerve dysfunction temporally associated with an outbreak of enterovirus D68 in children in Colorado, USA. Lancet 2015; 385(9978): 1662-1671.

12. Pastula DM, et al. Acute neurologic illness of unknown etiology in children - Colorado, August-September 2014. MMWR Morbidity and Mortality Weekly Report 2014; 63(40): 901-902.

13. European Centre for Disease Prevention and Control. Enterovirus 68 detected in the USA, Canada and Europe Second update, 25 November 2014. In. http:// ecdc.europa.eu/en/publications/Publications/

Enterovirus-68-detected-in-the-USA-Canada-Europe-second-update-25-November-2014.pdf, 2014.

14. Bragstad K, et al. High frequency of enterovirus D68 in children hospitalised with respiratory illness in Norway, autumn 2014. Influenza and Other Respiratory Viruses 2015; 9(2): 59-63.

15. Poelman R, et al. The emergence of enterovirus D68 in a Dutch University Medical Center and the necessity for routinely screening for respiratory viruses. Journal of Clinical Virology: the Official Publication of the Pan American Society for Clinical Virology 2015; 62: 1-5.

16. Public Health England. PHE National Polio Guidelines. Local and regional services. In. https://www.gov.uk/ government/uploads/system/uploads/attachment_data/ file/497601/PHE_Polio_guidelines.pdf:PublicHealth England, 2016.

17. Correa A, et al. Royal College of General Practitioners Research and Surveillance Centre (RCGP RSC) sentinel network: a cohort profile. BMJ Open 2016; 6(4): e011092.

18. Tamura K, et al. MEGA6: Molecular Evolutionary Genetics Analysis version 6.0. Molecular Biology and Evolution 2013; 30(12): 2725-2729.

19. Brown BA, et al. Seven strains of enterovirus D68 detected in the United States during the 2014 severe respiratory disease outbreak. Genome Announcements 2014; 2(6).

20. Wylie TN, et al. Development and evaluation of an enterovirus D68 real-time reverse transcriptase PCR assay. Journal of Clinical Microbiology 2015; 53(8): 2641-2647.

21. Kadambari S, et al. Enterovirus infections in England and Wales, 2000-2011: the impact of increased molecular diagnostics. Clinical Microbiology and Infection 2014; 20(12): 1289-1296.
22. Kaida A, et al. Enterovirus 68 in children with acute respiratory tract infections. Emerging Infectious Diseases 2011; 17(8): 1494-1497.

23. Smura TP, et al. Enterovirus 94, a proposed new serotype in human enterovirus species D. Journal of General Virology 2007; 88(PT 3): 849-858.

24. Prachayangprecha S, et al. Exploring the potential of next-generation sequencing in detection of respiratory viruses. Journal of Clinical Microbiology 2014; 52(10): 3722-3730.

25. Varghese R, et al. Sampling the upper respiratory tract for enteroviral infection is important in the investigation of an acute neurological illness in children. European Journal of Paediatric Neurology: EJPN: Official Journal of the European Paediatric Neurology Society 2015; 19(4): 494-495.

26. Tokarz R, et al. Worldwide emergence of multiple clades of enterovirus 68. The Journal of General Virology 2012; 93(Pt 9): 1952-1958.

27. Farrell JJ, et al. Enterovirus d68-associated acute respiratory distress syndrome in adult, United States, 2014. Emerging Infectious Diseases 2015; 21(5): 914-916.

28. Oberste MS, et al. Enterovirus 68 is associated with respiratory illness and shares biological features with both the enteroviruses and the rhinoviruses. The Journal of General Virology 2004; 85(Pt 9): 2577-2584.

29. Zhang T, et al. Enterovirus d68-associated severe pneumonia, china, 2014. Emerging Infectious Diseases 2015; 21(5): 916-918.

30. Reiche J, et al. Low-level circulation of enterovirus D68-associated acute respiratory infections, Germany, 2014. Emerging Infectious Diseases 2015; 21(5): 837-841.

31. Pfeiffer HC, et al. Two cases of acute severe flaccid myelitis associated with enterovirus D68 infection in children, Norway, autumn 2014. Euro Surveillance: Bulletin Europeen sur les maladies Transmissibles = European Communicable Disease Bulletin 2015; 20(10): 21062.

32. Lang M, et al. Acute flaccid paralysis following enterovirus D68 associated pneumonia, France, 2014. Eurosurveillance 2014; 19(44): pii. 20952.

33. Williams C, et al. Cluster of atypical adult Guillain-Barré syndrome temporally associated with neurological illness due to EV-D68 in children, South Wales, United Kingdom, October 2015 to January 2016. Eurosurveillance 2016; 21(4): pii. 30119.

34. Meijer A, et al. Continued seasonal circulation of enterovirus D68 in the Netherlands, 2011-2014. Eurosurveillance 2014; 19(42): pii. 20935.

35. Oberste MS, et al. Species-specific RT-PCR amplification of human enteroviruses: a tool for rapid species identification of uncharacterized enteroviruses. The Journal of General Virology 2006; 87(Pt 1): 119-128.

36. Centers for Disease Control and Prevention. Clusters of acute respiratory illness associated with human enterovirus 68-Asia, Europe, and United States, 20082010. MMWR Morbidity and Mortality Weekly Report 2011; 60(38): 1301-1304.

37. Poelman R, et al. European surveillance for enterovirus D68 during the emerging North-American outbreak in 
2014. Journal of Clinical Virology: the Official Publication of the Pan American Society for Clinical Virology 2015; 71: 1-9.

38. Li R, et al. Molecular analysis of virulent determinants of enterovirus 71. PLoS ONE 2011; 6(10): e26237.
39. Gromeier M, Alexander L, Wimmer E. Internal ribosomal entry site substitution eliminates neurovirulence in intergeneric poliovirus recombinants. Proceedings of the National Academy of Sciences of the United States of America 1996; 93(6): 2370-2375. 